

\title{
On the microscopic fluctuations driving the NMR relaxation of quadrupolar ions in water
}

Antoine Carof, Mathieu Salanne, Thibault Charpentier, Benjamin Rotenberg

\section{To cite this version:}

Antoine Carof, Mathieu Salanne, Thibault Charpentier, Benjamin Rotenberg. On the microscopic fluctuations driving the NMR relaxation of quadrupolar ions in water. Journal of Chemical Physics, 2015, 143, pp.194504. 10.1063/1.4935496 . cea-01230107

\section{HAL Id: cea-01230107 https://hal-cea.archives-ouvertes.fr/cea-01230107}

Submitted on 17 Nov 2015

HAL is a multi-disciplinary open access archive for the deposit and dissemination of scientific research documents, whether they are published or not. The documents may come from teaching and research institutions in France or abroad, or from public or private research centers.
L'archive ouverte pluridisciplinaire HAL, est destinée au dépôt et à la diffusion de documents scientifiques de niveau recherche, publiés ou non, émanant des établissements d'enseignement et de recherche français ou étrangers, des laboratoires publics ou privés. 


\section{AP $\mid \begin{aligned} & \text { The Journal of } \\ & \text { Chemical Physics }\end{aligned}$}

On the microscopic fluctuations driving the NMR relaxation of quadrupolar ions in water

Antoine Carof, Mathieu Salanne, Thibault Charpentier, and Benjamin Rotenberg

Citation: The Journal of Chemical Physics 143, 194504 (2015); doi: 10.1063/1.4935496

View online: http://dx.doi.org/10.1063/1.4935496

View Table of Contents: http://scitation.aip.org/content/aip/journal/jcp/143/19?ver=pdfcov

Published by the AIP Publishing

\section{Articles you may be interested in}

Solvation structure and transport properties of alkali cations in dimethyl sulfoxide under exogenous static electric fields

J. Chem. Phys. 142, 224502 (2015); 10.1063/1.4921982

Individual degrees of freedom and the solvation properties of water

J. Chem. Phys. 137, 024108 (2012); 10.1063/1.4732514

$1 \mathrm{H}$ NMR relaxation in glycerol solutions of nitroxide radicals: Effects of translational and rotational dynamics

J. Chem. Phys. 136, 114504 (2012); 10.1063/1.3692603

Two-particle random walk simulation of outer-sphere nuclear relaxation

J. Chem. Phys. 132, 224103 (2010); 10.1063/1.3429221

Solvation shell dynamics studied by molecular dynamics simulation in relation to the translational and rotational dynamics of supercritical water and benzene

J. Chem. Phys. 127, 174509 (2007); 10.1063/1.2780871

\section{AIP $\left.\right|_{\text {APL Photonics }}$}

APL Photonics is pleased to announce Benjamin Eggleton as its Editor-in-Chief

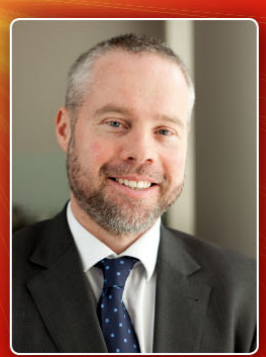




\title{
On the microscopic fluctuations driving the NMR relaxation of quadrupolar ions in water
}

\author{
Antoine Carof, ${ }^{1}$ Mathieu Salanne, ${ }^{1}$ Thibault Charpentier, ${ }^{2}$ and Benjamin Rotenberg ${ }^{1, a)}$ \\ ${ }^{1}$ Sorbonne Universités, UPMC Univ. Paris 06, CNRS, Laboratoire PHENIX, Case 51, 4 Place Jussieu, \\ F-75005 Paris, France \\ ${ }^{2}$ CEA, IRAMIS, NIMBE, LSDRM, UMR CEA-CNRS 3685, F-91191 Gif-sur-Yvette Cedex, France
}

(Received 24 September 2015; accepted 29 October 2015; published online 17 November 2015)

\begin{abstract}
Nuclear Magnetic Resonance (NMR) relaxation is sensitive to the local structure and dynamics around the probed nuclei. The Electric Field Gradient (EFG) is the key microscopic quantity to understand the NMR relaxation of quadrupolar ions, such as ${ }^{7} \mathrm{Li}^{+},{ }^{23} \mathrm{Na}^{+},{ }^{25} \mathrm{Mg}^{2+},{ }^{35} \mathrm{Cl}^{-},{ }^{39} \mathrm{~K}^{+}$, or ${ }^{133} \mathrm{Cs}^{+}$. Using molecular dynamics simulations, we investigate the statistical and dynamical properties of the EFG experienced by alkaline, alkaline Earth, and chloride ions at infinite dilution in water. Specifically, we analyze the effect of the ionic charge and size on the distribution of the EFG tensor and on the multi-step decay of its auto-correlation function. The main contribution to the NMR relaxation time arises from the slowest mode, with a characteristic time on the picosecond time scale. The first solvation shell of the ion plays a dominant role in the fluctuations of the EFG, all the more that the ion radius is small and its charge is large. We propose an analysis based on a simplified charge distribution around the ion, which demonstrates that the auto-correlation of the EFG, hence the NMR relaxation time, reflects primarily the collective translational motion of water molecules in the first solvation shell of the cations. Our findings provide a microscopic route to the quantitative interpretation of NMR relaxation measurements and open the way to the design of improved analytical theories for NMR relaxation for small ionic solutes, which should focus on water density fluctuations around the ion. (C) 2015 AIP Publishing LLC. [http://dx.doi.org/10.1063/1.4935496]
\end{abstract}

\section{INTRODUCTION}

Nuclear magnetic resonance (NMR) relaxation is a powerful tool to explore the structure and the dynamics of materials over a wide range of time and length scales. Extracting microscopic information from such experiments requires a microscopic model whose prediction for the relaxation rates is compared to the experimental data. For quadrupolar nuclei (with nuclear spin $I>1 / 2$ ), the main relaxation mechanism is the quadrupolar coupling between the electrostatic quadrupolar moment $e Q$ of the nucleus and the electric field gradient (EFG) at the site of the nucleus. Redfield theory provides the connection between the NMR relaxation rates and molecular motion, in terms of the following spectral density:

$$
J_{E F G}(\omega)=\int_{0}^{\infty}\left\langle V_{z z}(t) V_{z z}(0)\right\rangle e^{i \omega t} \mathrm{~d} t,
$$

where $V_{z z}$ is the $z z$ Cartesian component of the EFG, evaluated at multiples of the Larmor frequency $n \omega_{0}=n \gamma_{I} B_{0}$ (with $n \leq 2$ ) for the considered nucleus of gyromagnetic ratio $\gamma_{I}$ in a static magnetic field $B_{0}$, and brackets denote an ensemble-average. The spectral density is the Laplace Transform (taken at imaginary frequency $i \omega$ ) of the EFG auto-correlation function (EFG-ACF). In the case of simple molecular liquids, the EFG-ACF decays within a typical time $\tau \sim 1$ ps much shorter than the inverse of the Larmor frequency

\footnotetext{
a)benjamin.rotenberg@upmc.fr
}

$(>10 \mathrm{~ns})$. In this so-called extreme narrowing regime $\left(\omega_{0} \tau \ll 1\right)$, the transverse and longitudinal magnetizations relax exponentially with the characteristic rates, ${ }^{1,2}$

$$
\frac{1}{T_{1}}=\frac{1}{T_{2}}=\frac{3}{8} \frac{2 I+3}{I^{2}(2 I-1)}\left(\frac{e Q}{\hbar}\right)^{2} J_{E F G}(0) .
$$

Since the spin $I$ and the nuclear quadrupole $e Q$ are tabulated nucleus-specific properties, computing the relaxation rates then only requires the evaluation of spectral density (1) at zero frequency. Traditionally, relaxation processes in molecular liquids are interpreted by invoking Markovian assumptions on the translational and rotational dynamics of the nucleus of interest and of the surrounding medium, as well as treating the latter as a continuous polarizable background..$^{3-11}$

While such approximations may be in some cases relevant for large solutes, ignoring structural and dynamical features at the molecular scale is insufficient for smaller ones, such as simple ions or molecules. In these cases, resort to Monte Carlo and Molecular Dynamics (MD) simulations has provided a wealth of information on the EFG experienced by the considered nuclei. ${ }^{12-26}$ Some predictions of continuous theories were challenged by these results, such as the finding of a two-step decay of the EFG-ACF for $\mathrm{Li}^{+}, \mathrm{Na}^{+}$, and $\mathrm{Cl}^{-}$ or the evidence of the influence of symmetry in the first solvation shell on the EFG. We have recently shown for $\mathrm{Li}^{+}, \mathrm{Na}^{+}, \mathrm{K}^{+}$, and $\mathrm{Mg}^{2+}$ cations in water that a quantitative prediction of the relaxation rate can be obtained from classical 
MD simulations with an accurate polarizable force field, after properly connecting the exact EFG to the one induced by the classical charge distribution corresponding to the solvent around the ion. ${ }^{27}$

While molecular simulation provides to date the most accurate description of the structure and dynamics on the molecular scale, the large amount of raw information contained in the atomic trajectories should be thoughtfully interpreted in order to uncover the relevant processes. Here, we investigate using classical MD simulations the fluctuations of the EFG experienced by alkaline $\left(\mathrm{Li}^{+}, \mathrm{Na}^{+}, \mathrm{K}^{+}, \mathrm{Rb}^{+}\right.$, and $\left.\mathrm{Cs}^{+}\right)$and alkaline Earth $\left(\mathrm{Mg}^{2+}, \mathrm{Ca}^{2+}\right.$, and $\left.\mathrm{Sr}^{2+}\right)$ cations as well as the chloride $\mathrm{Cl}^{-}$anion in water at infinite dilution. We first quantify the statistical and dynamical features of these fluctuations, discussing in particular the effects of the ion radius and charge. We then analyze in more detail the microscopic origin of the observed properties and propose a simple model accounting for the fluctuations of the EFG, which demonstrates the essential role of water density fluctuations in the first solvation shell of the ions.

\section{METHODS}

\section{A. Theory}

In order to compute the relaxation rate using Eq. (2), it is necessary to evaluate the EFG-ACF, $J_{E F G}(0)$. To that end, one must overcome the challenge of simultaneously accurately predicting the EFG for any given configuration and simulating its dynamics over sufficiently long time scales to ensure the statistical convergence of the ACF. The former task requires in principle all electron ab initio simulations, but the entailed computational cost is to date not compatible with the latter constraint. Several approaches have been proposed to estimate the EFG in classical MD simulations, such as its computation on selected configurations from calculations at the Hartree-Fock ${ }^{28}$ and QM/MM ${ }^{25}$ levels, or the use of a function of positions fitted to prior ab initio computations on a simpler system. ${ }^{29}$ However, most classical MD studies rely on the so-called Sternheimer approximation, which postulates the linear response of the electronic cloud to the EFG arising from the "external" charge distribution of the classical molecular model describing the surrounding solvent. This leads to the following expression of the relaxation rate:

$$
\frac{1}{T_{1}}=\frac{1}{T_{2}}=\frac{3}{8} \frac{2 I+3}{I^{2}(2 I-1)}\left(\frac{e Q}{\hbar}\right)^{2}\left(1+\gamma_{\infty}\right)^{2} J(0),
$$

with $\gamma_{\infty}$ the Sternheimer factor and $J$ the spectral density of the "external" EFG. For the classical force field used in the present work, described below, we have recently shown the validity of the Sternheimer approximation in the case of the simple ions in water by comparing the classical external EFG to the $a b$ initio result computed at the Density Functional Theory (DFT) level with Projector Augmented Waves. ${ }^{27}$ This further allowed us to determine numerically the corresponding Sternheimer coefficients. Here, we focus only on the properties of the external EFG and the implications for the corresponding spectral density at zero frequency $J(0)$.

\section{B. Simulation details}

Alkaline $\left(\mathrm{Li}^{+}, \mathrm{Na}^{+}, \mathrm{K}^{+}, \mathrm{Rb}^{+}\right.$and $\left.\mathrm{Cs}^{+}\right)$and alkaline Earth $\left(\mathrm{Mg}^{2+}, \mathrm{Ca}^{2+}\right.$ and $\left.\mathrm{Sr}^{2+}\right)$ cations and the chloride $\mathrm{Cl}^{-}$anion in water at infinite dilution are modelled by a single ion and 215 water molecules in a cubic box of length $18.65 \AA$. We use a recently developed force field, based on the Polarizable Ion Model (PIM) ${ }^{30}$ in conjunction with the polarizable water model of Dang and Chang. ${ }^{31}$ The PIM is parametrized on DFT calculations in order to reproduce both the ab initio forces and dipoles and was shown to accurately describe the thermodynamic, structural, and dynamical properties of aqueous ions. ${ }^{30}$

Classical MD trajectories are generated using the $\mathrm{CP} 2 \mathrm{~K}$ simulation package. ${ }^{32}$ For each ion, 5 independent trajectories of $500 \mathrm{ps}$ in the $N V E$ ensemble are generated, obtained after annealing at $1000 \mathrm{~K}$ for $150 \mathrm{ps}$ followed by 50 ps equilibration at $298 \mathrm{~K}$, using a timestep of $1 \mathrm{fs}$. Periodic boundary conditions in all directions are used. A cutoff of $9.325 \AA$ is used for shortrange interactions, while electrostatic interactions and the EFG are computed with Ewald summation. ${ }^{33}$ Water molecules are treated as rigid using the SHAKE algorithm. ${ }^{34,35}$ During equilibration the temperature is maintained at the target value using a Nosé-Hoover thermostat ${ }^{36,37}$ with a time constant of 1 ps. The EFG-ACF is computed from the EFG every sampled every 5 fs along the trajectories. Error bars indicate the standard deviation among the 5 independent trajectories.

\section{RESULTS AND DISCUSSION}

\section{A. Statistical properties}

We begin our examination of the properties of the EFG tensor induced by the external charge distribution around the ions by considering its statistical distribution. In principle, it is fully characterized by the joint probability $P\left(V_{x x}, V_{x y}, V_{x z}, V_{y x}, V_{y y}, V_{y z}, V_{x z}, V_{y z}, V_{z z}\right)$ of its 9 Cartesian components. However, the EFG is a symmetric and traceless tensor, so that only 5 components are independent. In addition, the considered systems are rotationally invariant and it is common to define the 5 spherical components as follows: ${ }^{38,39}$

$$
\begin{aligned}
& U_{1}=V_{z z} / 2, \\
& U_{2}=V_{x z} / \sqrt{3}, \\
& U_{3}=V_{y z} / \sqrt{3}, \\
& U_{4}=V_{x y} / \sqrt{3}, \\
& U_{5}=\left(V_{x x}-V_{y y}\right) / 2 \sqrt{3} .
\end{aligned}
$$

It is therefore sufficient to consider the joint distribution $P\left(U_{1}, U_{2}, U_{3}, U_{4}, U_{5}\right)$, which should satisfy the following properties: ${ }^{39}$ (1) $\left\langle U_{i}\right\rangle=0$ for $i=1 \ldots 5$; (2) $\left\langle U_{i} U_{j}\right\rangle=\sigma_{c}^{2} \delta_{i j}$ for $i, j=1 \ldots 5$ with the same variance $\sigma_{c}^{2}$ for all components; (3) the four marginal distributions $P\left(U_{i}\right)$ for $i=2 \ldots 5$ are identical.

Figure 1 shows the marginal distributions $P\left(U_{i}\right)$ for $i=1 \ldots 5$ for the potassium ion. Not only are the 4 last distributions identical as expected for an isotropic system, but $U_{1}$ is also identical to the others. It follows from 


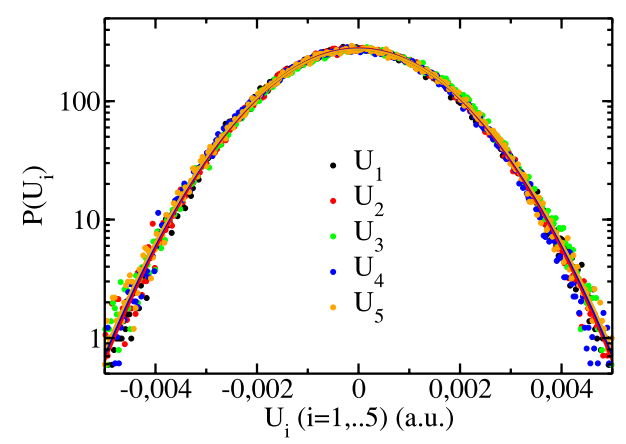

FIG. 1. Distribution of the 5 spherical components $U_{i}$ (see Eq. (4)) of the Electric Field Gradient (EFG) experienced by a potassium ion in water (in atomic units). For each spherical component, the Gaussian distribution with the same variance is shown (lines).


FIG. 2. Distribution $P\left(U_{1}\right)$ of the first spherical component of the EFG (in atomic units) at the site of an ion in a infinite dilute electrolyte (symbols): (a) alkali ions and (b) alkaline Earth and chloride ions. For each ion, the Gaussian distribution with the same variance are shown (lines).

this observation that all 5 variances $\left\langle U_{i}^{2}\right\rangle=\sigma_{c}^{2}$ are equal. In addition, it is clear from the parabolic shape on a logarithmic scale that this distribution is Gaussian. It further has a vanishing average. All these results also apply to the other ions considered here, so that it is sufficient to analyze the distribution for only one of the spherical components, illustrated in Figure 2, which also reports the Gaussian distributions with the same variance as the MD data.

Table I indicates the variance of the external EFG, which is the initial value of the EFG-ACF and is simply obtained from the common variance of the marginal distributions

TABLE I. Variance $\left\langle\mathbf{V}^{2}\right\rangle$ of the electric field gradient (EFG) at the nucleus for the spherical components $U_{i}$ of the EFG (see Eq. (4)). For each ion, average values and standard deviations correspond to 5 independent trajectories.

\begin{tabular}{cc}
\hline \hline Ion & $\left\langle\mathbf{V}^{2}\right\rangle\left(10^{38}\right.$ S.I. $)$ \\
\hline $\mathrm{Li}^{+}$ & $521 \pm 10$ \\
$\mathrm{Na}^{+}$ & $109 \pm 2$ \\
$\mathrm{~K}^{+}$ & $58.5 \pm 0.6$ \\
$\mathrm{Rb}^{+}$ & $41.2 \pm 0.5$ \\
$\mathrm{Cs}^{+}$ & $27.3 \pm 0.3$ \\
$\mathrm{Mg}^{2+}$ & $150 \pm 5$ \\
$\mathrm{Ca}^{2+}$ & $72.7 \pm 0.8$ \\
$\mathrm{Sr}^{2+}$ & $60.1 \pm 1$ \\
$\mathrm{Cl}^{-}$ & $154 \pm 2$ \\
\hline \hline
\end{tabular}

$P\left(U_{i}\right)$ as $\left\langle\mathbf{V}^{2}\right\rangle=\sum_{\alpha, \beta}\left\langle V_{\alpha \beta}^{2}\right\rangle=6 \sum_{i=1}^{5}\left\langle U_{i}^{2}\right\rangle=30 \sigma_{c}^{2}$. A more quantitative assessment of the Gaussian character of these distributions can be obtained by computing the normalized kurtosis $\gamma_{2}=\left\langle U_{i}^{4}\right\rangle /\left\langle U_{i}^{2}\right\rangle^{2}-3$. The normalized kurtosis is only of a few $\%$ for most ions, confirming the Gaussian behaviour. The largest deviations are observed for the smaller ions and the more highly charged ones $\left(\gamma_{2}=14 \%, 8 \%\right.$, and $37 \%$ for $\mathrm{Li}^{+}, \mathrm{Na}^{+}$, and $\mathrm{Mg}^{2+}$, respectively).

Both Figure 2 and Table I show that the variance of the external EFG decreases with increasing ion radius for a given ion charge (from $\mathrm{Li}^{+}$to $\mathrm{Cs}^{+}$and from $\mathrm{Mg}^{2+}$ to $\mathrm{Sr}^{2+}$ ). In addition, in a given row of the periodic table $\left\langle\mathbf{V}^{2}\right\rangle$ increases with the ionic charge (from $\mathrm{Na}^{+}$to $\mathrm{Mg}^{2+}, \mathrm{K}^{+}$to $\mathrm{Ca}^{2+}$ and $\mathrm{Rb}^{+}$ to $\mathrm{Sr}^{2+}$ ). This can be understood by noticing that the EFG due to a water molecule is stronger when its distance $r$ to the ion is shorter: For a radially oriented dipole, it scales as $\sim r^{-4}$. This distance, for water molecules in the first solvation shell of the ion, increases with ion radius and decreases with increasing ion charge (radial distribution functions for the force field used in the present work can be found in Ref. 30). Finally, comparing the isoelectronic $\mathrm{K}^{+}$and $\mathrm{Cl}^{-}$, we observe an asymmetry upon charge inversion, due to the asymmetry of the charge distribution on the water molecule, as discussed below. Higher order electrostatic multipoles of the water molecule are known to play a role in the solvation of ions, in particular, on their hydration free energy. ${ }^{40}$

Such a Gaussian behaviour has already been reported for other electrostatic quantities. The Gaussian fluctuations of the electrostatic potential on an ion are at the basis of Marcus' theory of electron transfer in solution ${ }^{41,42}$ and it has been confirmed in DFT-based ab initio MD. ${ }^{43}$ The fluctuations of the electric field in liquid water have also been investigated: While the field experienced by a proton displays non-Gaussian features which can be evidenced by IR spectroscopy ${ }^{44,45}$ and its rare fluctuations are at the origin of water autoionization, ${ }^{46,47}$ the statistics of the field experienced by ions are Gaussian in the bulk liquid. Its fluctuations play a role in the behaviour of ions near the liquid-vapour interface ${ }^{48}$ and in the mechanism of ionic pair dissociation. ${ }^{49}$ It should be noted that, while the Gaussian behaviour extends to relatively large deviations from the mean (at least 3 standard deviations), the present equilibrium simulations only probe fluctuations around the mean and that advanced sampling techniques would be required to probe rare fluctuations.

In contrast, the statistics of the EFG on the oxygen and hydrogen of water are not Gaussian, as a result of the local anisotropy of the charge distribution (see the supplementary material ${ }^{50}$ ). The fact that the components of the EFG experienced by the ions follow a Gaussian statistics, while not surprizing from the known behaviour of the electrostatic potential and of the electric field, is not trivial since these quantities do not scale similarly with the distance to the ion. Therefore individual molecules in a given configuration contribute differently to the various observables, which in turn obey different statistics. As an illustration, Figure 3 reports the statistics of the force on the ion, which displays fat tails (leptokurtic behaviour) arising from the short-range binary collisions with the closest molecule - a feature analyzed in detail in the case of simple fluids..$^{51,52}$ 

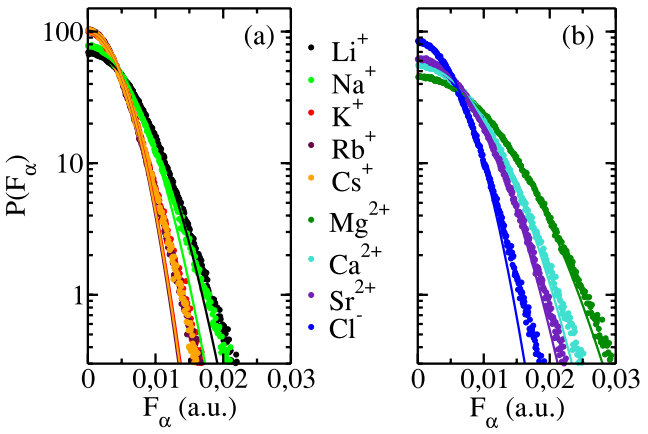

FIG. 3. Distribution of the Cartesian components of the force $(\alpha=x, y, z)$ on alkaline (a), alkaline Earth and chloride (b) ions at infinite dilution in water. For each ion, the Gaussian distribution with same variance is also shown to highlight the leptokurtic behaviour of the force distribution.

The fact that all marginal distributions of the spherical components are equal and Gaussian suggests that the EFG tensor itself may follow the statistics of the Gaussian Isotropic Model (GIM) developed by Czjzek et al., ${ }^{38,39,53}$

$$
P_{G I M}\left(U_{1}, U_{2}, U_{3}, U_{4}, U_{5}\right)=\prod_{i=1}^{5} \frac{e^{-U_{i}^{2} / 2 \sigma_{c}^{2}}}{\sqrt{2 \pi \sigma_{c}^{2}}} .
$$

Such a model not only implies the equality of the marginal distributions but further requires the absence of correlations between the 5 spherical components. This stronger constraint can be tested in the present case by analyzing the statistics of the eigenvalues of the EFG tensor. Indeed, under the assumptions of the GIM, the largest positive eigenvalue $(v>0)$ tensor should be distributed according to

$$
\begin{aligned}
P(v)= & \frac{1}{\sigma_{c}} \sqrt{\frac{2}{\pi}}\left[\left(\frac{3 v^{2}}{8 \sigma_{c}^{2}}-1\right) e^{-v^{2} / 8 \sigma_{c}^{2}}\right. \\
& \left.+\left(1-\frac{v^{2}}{3 \sigma_{c}^{2}}\right) e^{-v^{2} / 6 \sigma_{c}^{2}}\right] .
\end{aligned}
$$

Figure 4 shows the marginal distributions of the three eigenvalues of the EFG felt by a potassium ion, together with the prediction of the GIM. While not perfect, the agreement with Eq. (6) is very good. It suggests that correlations between the spherical components may be limited. In turn, this implies some correlations between the Cartesian components of the EFG, which reflect the local structure of the solvent around the ions. Nevertheless, the above discussion of the effects of



FIG. 4. Distribution of the three eigenvalues $\left(v_{1}>v_{2}>v_{3}\right)$ of the EFG at the site of a potassium ion in water. The analytical result for the largest eigenvalue in the Gaussian Isotropic Model of Czjzek, Eq. (6) is also shown (blue line). ion radius and charge on the variance, which captures the simulation results by accounting only for the bare effect of the distance from the ion and neglecting symmetry effects, suggests that the former may play the dominant role in the present case.

\section{B. Dynamics of EFG fluctuations}

We now turn to the dynamical side of EFG fluctuations and analyze the normalized ACF,

$$
C_{E F G}^{n o r m}(\tau)=\frac{\langle\mathbf{V}(\tau): \mathbf{V}\rangle}{\left\langle\mathbf{V}^{2}\right\rangle}=\frac{1}{\left\langle\mathbf{V}^{2}\right\rangle} \sum_{\alpha, \beta}\left\langle V_{\alpha \beta}(\tau) V_{\alpha \beta}\right\rangle
$$

and its running integral

$$
I_{E F G}^{n o r m}(\tau)=\int_{0}^{\tau} C_{E F G}^{n o r m}(t) \mathrm{d} t .
$$

Both quantities are shown in Figure 5 for the ions considered in the present work. For all ions, the normalized ACF decays to zero within a few ps. This decay occurs in a non-trivial way, suggesting that several processes are at play. Such a multistep was already reported by Engström and Jönsson, ${ }^{15}$ Roberts and Schnitker, ${ }^{16}$ and Odelius. ${ }^{18}$ Importantly, this observation invalidates simple continuous theories usually invoked to interpret the NMR relaxation of aqueous ions, which predict a mono-exponential decay with a single characteristic time scale. However, the molecular mechanisms leading to the observed multi-step decay remain to be clarified. To that end, we first analyze quantitatively the EFG-ACF.
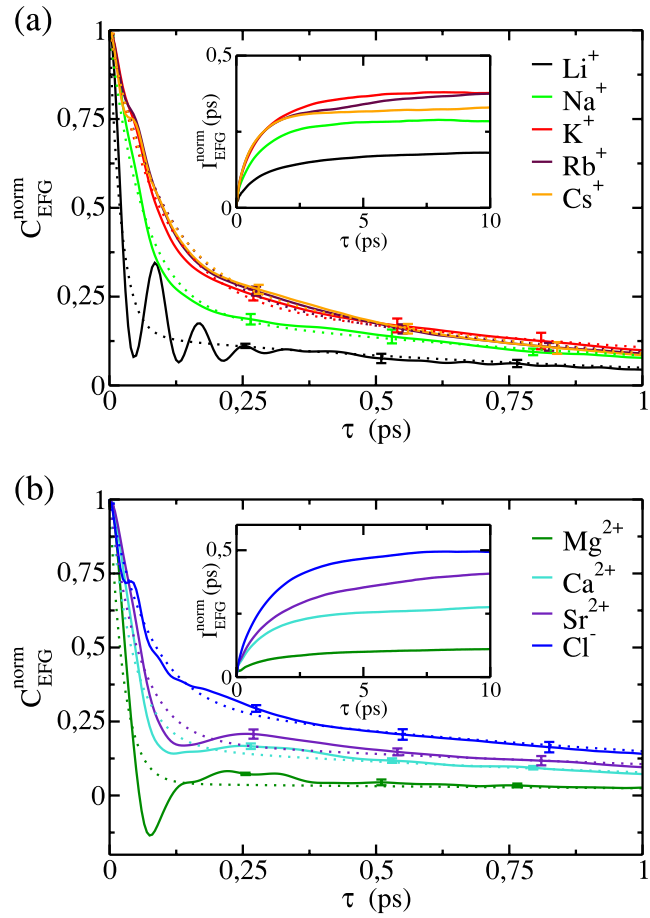

FIG. 5. Normalized correlation functions $C_{E F G}^{n o r m}$ and their integrals (inserts) of the EFG at the nucleus site of different ions at infinite dilution in water: (a) alkali ions (b) alkaline Earth and chloride ions. Error bars indicate standard deviations over 5 independent trajectories, while dotted lines correspond to a fit of the data to Eq. (9). 


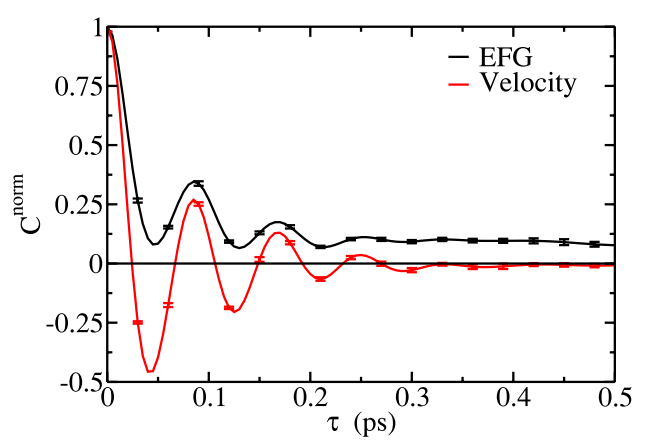

FIG. 6. Normalized correlation functions for the aqueous lithium ion: EFG at the site of the nucleus (black) and velocity (red). Error bars indicate standard deviations over 5 independent trajectories.

While the decay is monotonic for most ions, the EFG-ACF displays oscillations for lithium and magnesium. In the $\mathrm{Li}^{+}$case, the origin of these oscillations can be assessed by comparing the auto-correlation of the EFG to that of the ion velocity (VACF). Both normalized correlation functions are reported in Figure 6. Both these correlation functions oscillate in-phase at short times. This striking result can be traced back to the smaller mass of the ion $(7 \mathrm{~g} / \mathrm{mol})$ compared to water molecules $(18 \mathrm{~g} / \mathrm{mol})$. Due to the larger inertia of the solvent, the motion of $\mathrm{Li}^{+}$can be thought of, over a short lapse of time ( $200 \mathrm{fs})$, as one in a frozen external potential. As the $\mathrm{Li}^{+}$ion oscillates in this potential, it runs through the EFG hypersurface and consequently the EFG-ACF oscillates in phase with the VACF.

For all ions (leaving now aside the oscillations for $\mathrm{Li}^{+}$and $\mathrm{Mg}^{2+}$ ), the EFG-ACF decays approximately in two steps, with a fast and substantial decrease within a short characteristic time $\tau_{f} \sim 100$ fs and a slower second regime with a longer characteristic time $\tau_{s} \sim 1 \mathrm{ps}$, corresponding to a fraction $\alpha_{s} \sim 20 \%-30 \%$ of the decay. More precisely, in the absence of a definite molecular mechanism to interpret the data at this stage, we fit the normalized EFG-ACF by two exponentials corresponding to the above-mentioned two steps,

$$
C_{f i t}^{n o r m}(\tau)=\left(1-\alpha_{s}\right) \mathrm{e}^{-\tau / \tau_{f}}+\alpha_{s} \mathrm{e}^{-\tau / \tau_{s}} .
$$

The resulting characteristic times and amplitudes are summarized in Table II, together with the integral of the EFG-ACF $J(0)$ and the fraction of this integral arising from the slow contribution obtained by integrating the corresponding part in Eq. (9): $J_{s}(0)=\left\langle\mathbf{V}^{2}\right\rangle \alpha_{s} \tau_{s}$.

The characteristic time $\tau_{f}$ for the fast process increases when going down in a given column of the periodic table (except for $\mathrm{Cs}^{+}$), while it decreases (by a factor of $\sim 2$ ) along rows. In contrast, the characteristic time $\tau_{s}$ for the slow process decreases along the alkaline Earth series (from $\sim 2 \mathrm{ps}$ for $\mathrm{Mg}^{2+}$ to $\sim 1.2 \mathrm{ps}$ for $\mathrm{Sr}^{2+}$ ) and displays a non-monotonic behaviour along the alkaline series, with a maximum for $\mathrm{K}^{+}$, similar to that already reported for the mobility of these ions in water. ${ }^{54,55}$ For the only considered anion, $\mathrm{Cl}^{-}$, the short time $\tau_{f}$ is comparable to that of alkaline cations, while the long time $\tau_{s}$ is closer to that of alkaline Earth cations.

The weight $\alpha_{s}$ of the slow process in the EFG-ACF increases along columns and decreases along rows of the periodic table. It remains small for all ions and ranges from
TABLE II. Characteristic times for the fast $\left(\tau_{f}\right)$ and slow $\left(\tau_{s}\right)$ decay of the EFG, fraction $\alpha_{s}$ of the decay arising from the slow mode, spectral density at zero frequency $J(0)$ and fraction of the latter due to the slow decay, $J_{s}(0) / J(0)=\left\langle\mathbf{V}^{2}\right\rangle \alpha_{s} \tau_{s} / J(0)$.

\begin{tabular}{lccccc}
\hline \hline Ion & $\begin{array}{c}\tau_{f} \\
(\mathrm{fs})\end{array}$ & $\begin{array}{c}\tau_{s} \\
(\mathrm{fs})\end{array}$ & $\begin{array}{c}\alpha_{s} \\
(\%)\end{array}$ & $\begin{array}{c}J(0) \\
\left(10^{27} \mathrm{~S} . \mathrm{I} .\right)\end{array}$ & $\begin{array}{c}J_{S}(0) / J(0) \\
(\%)\end{array}$ \\
\hline $\mathrm{Li}^{+}$ & 22 & 950 & 14 & 9.37 & 75 \\
$\mathrm{Na}^{+}$ & 63 & 1070 & 21 & 3.11 & 81 \\
$\mathrm{~K}^{+}$ & 91 & 1140 & 26 & 2.19 & 79 \\
$\mathrm{Rb}^{+}$ & 97 & 900 & 29 & 1.54 & 69 \\
$\mathrm{Cs}^{+}$ & 86 & 760 & 34 & 0.90 & 78 \\
$\mathrm{Mg}^{2+}$ & 26 & 1990 & 4 & 1.65 & 72 \\
$\mathrm{Ca}^{2+}$ & 45 & 1300 & 17 & 2.0 & 80 \\
$\mathrm{Sr}^{2+}$ & 55 & 1180 & 19 & 2.44 & 54 \\
$\mathrm{Cl}^{-}$ & 74 & 1370 & 31 & 7.57 & 87 \\
\hline \hline
\end{tabular}

$4 \%$ for $\mathrm{Mg}^{2+}$ to $34 \%$ for $\mathrm{Cs}^{+}$. Nevertheless, the slow process accounts for most of the contribution to the integral $J(0)$, which is the quantity appearing in expression (2) of the relaxation time. Indeed, the ratio $J_{s}(0) / J(0)$ (for which no clear trend emerges from Table II) is usually larger than $70 \%$, with the exception of $\mathrm{Sr}^{2+}(54 \%)$.

The above analysis suggests that the evolution of $J(0)$ $=\left\langle\mathbf{V}^{2}\right\rangle \lim _{\tau \rightarrow \infty} I_{E F G}^{n o r m}(\tau)$ can be rationalized by considering the effects on the one hand of $\left\langle\mathbf{V}^{2}\right\rangle$, already discussed in Sec. III A (see Table I), and on the other hand on the product $\alpha_{s} \tau_{s}$. In particular, $J(0)$ decreases by a factor of 10 from $\mathrm{Li}^{+}$to $\mathrm{Cs}^{+}$, while it increases by $\sim 50 \%$ from $\mathrm{Mg}^{2+}$ to $\mathrm{Sr}^{2+}$. This should be compared to the decrease in $\left\langle\mathbf{V}^{2}\right\rangle$ for both series, by a factor of $\sim 20$ for the alkaline ions and 2.5 for the alkaline Earth ions (see Table I). Given the moderate variations of $\tau_{s}$ compared to that of $\alpha_{s}$ and $\left\langle\mathbf{V}^{2}\right\rangle$, we can conclude that the evolution of $J(0)$ is dominated by the competition between these last two terms. While at this stage it is impossible to assess the microscopic origin of the two-step decay, we can hypothesize that the two regimes arise from distinct processes, each contributing to the variance $\left\langle\mathbf{V}^{2}\right\rangle$. Both contributions decrease with increasing ionic size, but the one corresponding to the slow process decreasing less than the other one.

We conclude this quantitative study of the dynamical fluctuations of the EFG by mentioning that at very long times a mode-coupling theory of Bosse et al. predicts an algebraic decay (as $t^{-5 / 2}$ ) for the EFG-ACF. ${ }^{56}$ Such a decay should arise from the hydrodynamic fluctuations of the solvent, which result in particular in the well-known $t^{-3 / 2}$ scaling of the velocity auto-correlation function. ${ }^{57}$ However, the effect of these fluctuations can only manifest themselves for sufficiently large systems and time scales covering several orders of magnitude, so that we could not identify such a scaling in our simulations (results on a logarithmic scale not shown).

\section{First solvation shell}

Finally, we examine the microscopic origin of the fluctuations of the EFG. Specifically, we aim at identifying the main contribution to the EFG in the charge distribution from which it arises. In the discussion of the statistical properties 
of the EFG, it already appeared that the EFG is a rather short-ranged quantity. This suggests to determine, as done previously by others, ${ }^{12,13,15}$ the contribution of molecules closest to the ions, i.e., in their first coordination shell. The latter can be defined from the radial distribution function and we refer the reader to Ref. 30 for the present model of ions at infinite dilution. Therefore, we investigate here the EFG computed for each configuration by considering only the water molecules in its first solvation shell (Solvent Shell Model, SSM).

In addition, the charge distribution associated with these few molecules may be further simplified, by replacing the partial charges and the induced dipole of the Dang-Chang water model by a single radial dipole identical for all molecules, placed on the oxygen atom, of norm $\mu_{r}^{\text {eff }}$ to be determined. This Radial Dipole Model (RDM), schematically illustrated in the inset of Figure 7, is thus a very crude approximation of the charge distribution. The corresponding $\mathrm{EFG}$ at the site of the ion reads

$$
\mathbf{V}^{R D M}=3 \mu_{r}^{e f f} \mathbf{S}^{R D M}=3 \mu_{r}^{e f f} \sum_{i \in \mathcal{S}} \frac{3 \mathbf{r}_{i} \otimes \mathbf{r}_{i}-r_{i}^{2} \mathbf{I}}{r_{i}^{6}},
$$

where the sum defining the tensor $\mathbf{S}^{R D M}$ runs over oxygen atoms $i$ in the first solvation shell $\mathcal{S}$, at positions $\mathbf{r}_{i}$ relative to the ion, and where $\mathbf{I}$ is the identity tensor. In particular, changes in $\mathbf{V}^{R D M}$ reflect only the translational dynamics of water within the first solvation shell.

Figure 7 shows the Cartesian components of the exact EFG $\mathbf{V}$ as a function of that of the two simple models just described: $\mathbf{V}^{S S M}$ and $\mathbf{S}^{R D M}$. In both cases, a linear relation is found. The corresponding slopes $k^{S S M}$ and $k^{R D M}=3 \mu_{r}^{e f f}$ are reported in Table III, together with the relative errors,

$$
\sigma^{X}(\mathbf{V})=\sqrt{\frac{\left\langle\delta \mathbf{V}^{2}\right\rangle}{\left\langle\mathbf{V}^{2}\right\rangle}}=\sqrt{\frac{\frac{1}{N} \sum_{i=1}^{N}\left|V_{i}-k^{X} V_{i}^{X}\right|^{2}}{\frac{1}{N} \sum_{i=1}^{N}\left|V_{i}\right|^{2}}},
$$

where the sum runs over the Cartesian components of the EFG tensor for $10^{4}$ configurations. This quantity allows to measure

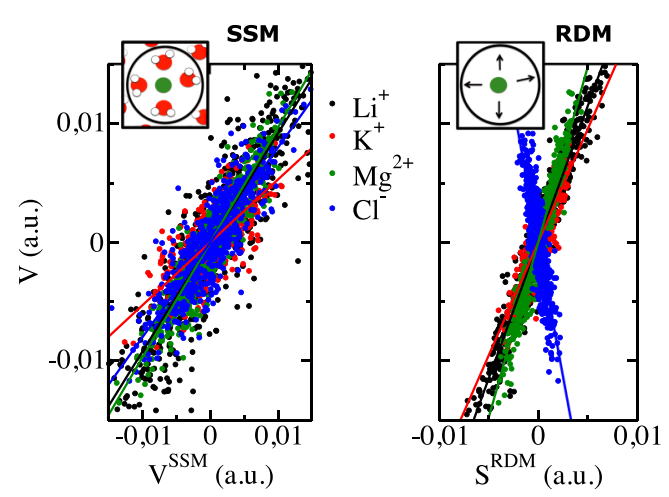

FIG. 7. Correlation between the exact EFG $\mathbf{V}$ and two models calculated from restricted charge distributions, corresponding to the first solvation shell only (SSM, left) and to the radial dipole model (RDM, right). For the latter, the EFG is reported as a function of the $\mathbf{S}^{R D M}$ tensor defined by Eq. (10). The inserts depict the two models. The slopes of the linear regression, $k^{S S M}$ and $k^{R D M}=3 \mu_{r}^{e f f}$ are given in Table III. For each ion, 6 Cartesian components of the EFG (in atomic units) are shown for 100 configurations.
TABLE III. Proportionality coefficient $k$ between the exact EFG $\mathbf{V}$ and two EFGs calculated from restricted charge distributions, corresponding to the first solvation shell only (SSM) and to the radial dipole model (RDM, see text). For the RDM, the slope is given in terms of the effective radial dipole $\mu_{r}^{e f f}=k^{R D M} / 3$ and compared to the average radial dipole from the full atomistic model, $\left\langle\mu_{r}\right\rangle$. Errors on the slopes are smaller than $1 \%$. The errors between the two model EFGs relative to the exact one, $\sigma(\mathbf{V})$ (see Eq. (11)), are also indicated.

\begin{tabular}{lccccc}
\hline \hline Ions & $k^{S S M}$ & $\sigma^{S S M}(\mathbf{V})$ & $\begin{array}{c}\mu_{r}^{\text {eff }}=k^{R D M} / 3 \\
\text { (a.u.) }\end{array}$ & $\sigma^{R D M}(\mathbf{V})$ & $\begin{array}{r}\left\langle\mu_{r}\right\rangle \\
\text { (a.u.) }\end{array}$ \\
\hline $\mathrm{Li}^{+}$ & 0.92 & 0.42 & 0.77 & 0.56 & 0.89 \\
$\mathrm{Na}^{+}$ & 0.64 & 0.77 & 0.63 & 0.62 & 0.58 \\
$\mathrm{~K}^{+}$ & 0.53 & 0.94 & 0.64 & 0.64 & 0.54 \\
$\mathrm{Rb}^{+}$ & 0.47 & 1.06 & 0.60 & 0.68 & 0.47 \\
$\mathrm{Cs}^{+}$ & 0.43 & 1.12 & 0.47 & 0.77 & 0.41 \\
$\mathrm{Mg}^{2+}$ & 0.97 & 0.37 & 0.99 & 0.70 & 1.16 \\
$\mathrm{Ca}^{2+}$ & 0.80 & 0.55 & 0.89 & 0.68 & 0.97 \\
$\mathrm{Sr}^{2+}$ & 0.49 & 1.0 & 0.87 & 0.67 & 0.92 \\
$\mathrm{Cl}^{-}$ & 0.80 & 0.55 & -1.52 & 1.16 & -0.67 \\
\hline \hline
\end{tabular}

the accuracy of the models $X=S S M$ and $R D M$ describing the contribution of the first solvation shell.

Despite the simplicity of both models, the linear correlation confirms that the first solvation shell plays an important role on the EFG at the nucleus site. Considering first the full charge distribution of these molecules (SSM), we observe that the slope for $\mathrm{Li}^{+}$and $\mathrm{Mg}^{2+}$ is close to unity, with the smallest relative errors $(40 \%)$ between the contribution of molecules in the first solvation shell and the total EFG. For a given ion size the contribution of molecules beyond the first solvation shell is less important for more highly charged ions For isovalent ions, the slope decreases with increasing ionic radius and the correlation between the SSM and exact EFG deteriorates. Nevertheless, the fact that a linear correlation remains suggests that it may be possible to encompass the contribution of molecules beyond the first solvation shell within the framework of a polarizable continuum. Conversely, one may also consider the response of the first solvation shell as resulting from the competition between its polarization by the fluctuating polarizable continuum around $\mathrm{it}^{58}$ and that by the electric field of the ion, as well as more molecular packing and orientational effects. The stronger the field of the ion, i.e., larger charge or smaller radius, the less important the impact of the surrounding solvent on the structure of the first solvation shell - hence on the EFG experienced by the ion. To our knowledge, the implications of the now standard discussion of specific ionic effects on the structure of water ("kosmotrope" and "chaotrope" ions) $)^{59-61}$ on the EFG and NMR relaxation of ions had not been considered previously.

We now turn to the even simpler RDM. The linear correlation between the EFG and the $\mathbf{S}^{R D M}$ tensor may be all the more surprizing that the associated error is smaller for most ions than with the SSM model, which describes the full charge distribution in the first solvation shell. However, one should keep in mind that the slope $k^{R D M}=3 \mu_{r}^{e f f}$ is fitted to minimize this error, whereas the slope $k^{S S M}$ is not an adjustable parameter. The fact that the slope $k^{R D M}$ is in the range 1.5-3 


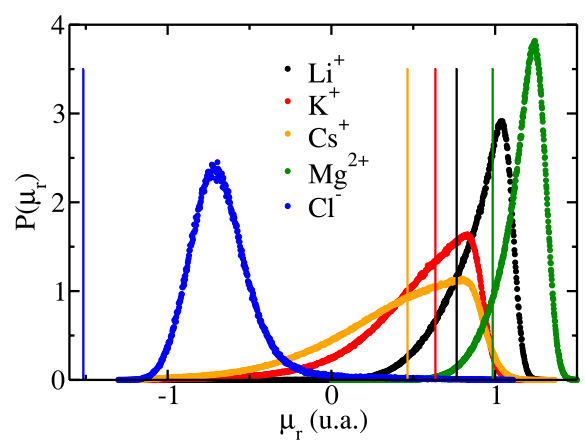

FIG. 8. Distribution of the radial component $\mu_{r}$ of the dipole of water molecules in the first solvation shell, including the induced dipole (in atomic units), for selected ions. Vertical lines indicate the value of the effective radial dipole $\mu_{r}^{e f f}$ of the radial dipole model.

(or -4.5 in the case of $\mathrm{Cl}^{-}$, discussed below) indicates that several (possibly competing) effects are lumped together into the effective radial dipole $\mu_{r}^{e f f}$, such as the orientation of each water molecule, its multipolar distribution and its polarization by the local electric field, or even the effect of molecules beyond the first solvation shell. Nevertheless the predictions of this extremely simple model are in good agreement with the total EFG.

In order to further assess its physical relevance, we compare $\mu_{r}^{e f f}$ to the average radial dipole $\left\langle\mu_{r}\right\rangle$ of water molecules in the first solvation shell, also reported in Table III. For all cations, the effective radial dipole is in good agreement with the average one (within 10\%-20\%) confirming the relevance of the RDM in this case. As an illustration, Figure 8 reports the distribution of the radial component of the dipole of water molecules in the first solvation shell of various ions (including the induced dipole), together with the corresponding $\mu_{r}^{e f f}$. In contrast, for the chloride anion, despite the expected negative sign of the $\mu_{r}^{e f f}$, the magnitude differs by a factor $\sim 2.2$ from the average one. In fact, it does not even fall within the range of values sampled during the simulation (see Figure 8). Such a failure for this anion is consistent with the fact that the water molecules in the first solvation shell are not radially oriented but rather tend to donate a hydrogen bond to the anion. ${ }^{62}$

The good performance of the RDM for cations and the physical relevance of the corresponding effective radial dipole suggest that the EFG experienced by these ions depends primarily on the position of the water molecules around it. Indeed, this simple model relies only on the positions of the oxygen atoms and not on their orientation. The simple $\mathbf{S}^{R D M}$ tensor is sufficient to capture the effect of the structure of the first solvation shell, including the collective symmetry effects relevant to the EFG. The latter have been shown to play an important role on the $\mathrm{EFG}^{16}$ and indeed we could not correlate the EFG with any quantity (position or orientation) associated with single molecules.

Overall, the above discussion indicates that the EFG-ACF reflects the collective translational motion of water molecules in the first solvation shell of the cations. We finally consider the implications of such an assertion on the NMR relaxation rate by computing the spectral density at zero-frequency $J(0)$ within the SSM and RDM models. Results are summarized
TABLE IV. Spectral density at zero frequency $J(0)$ computed using the exact EFG and two EFGs calculated from restricted charge distributions, corresponding to the first solvation shell (SSM) and to the radial dipole model (RDM, see text).

\begin{tabular}{llll}
\hline \hline & & $J(0)\left(10^{27}\right.$ S.I. $)$ & \\
\cline { 2 - 4 } Ions & Exact & SSM & RDM \\
\hline $\mathrm{Li}^{+}$ & 9.37 & 6.91 & 9.79 \\
$\mathrm{Na}^{+}$ & 3.11 & 4.40 & 2.46 \\
$\mathrm{~K}^{+}$ & 2.19 & 2.43 & 1.86 \\
$\mathrm{Rb}^{+}$ & 1.54 & 1.77 & 1.07 \\
$\mathrm{Cs}^{+}$ & 0.90 & 0.94 & 0.47 \\
$\mathrm{Mg}^{2+}$ & 1.65 & 2.04 & 1.29 \\
$\mathrm{Ca}^{2+}$ & 2.00 & 1.67 & 1.79 \\
$\mathrm{Sr}^{2+}$ & 2.44 & 2.76 & 2.22 \\
$\mathrm{Cl}^{-}$ & 7.57 & 5.0 & 6.78 \\
\hline \hline
\end{tabular}

in Table IV. While not quantitative, the predictions of both models are in relatively good agreement with the exact result, in particular, they provide the correct order of magnitude and capture the trends along the columns and rows of the periodic table (excepted for calcium within the SSM). Importantly, the RDM provides an even better estimate despite its coarser description of the charge distribution giving rise to the EFG. As explained above, this is due to the fact the use of an effective radial dipole as an adjustable parameter, even physically relevant since it follows the average radial dipole, accounts for several effects simultaneously. Nevertheless, the good predictions of the RDM for the spectral density at zero-frequency $J(0)$ underline that the dynamical fluctuations of the EFG experienced by cations can be well described by a model involving only the collective translational motion of water molecules in their first solvation shell.

\section{CONCLUSION}

Using molecular dynamics simulations, we have investigated the statistical and dynamical properties of the electric field gradient,. experienced by alkaline, alkaline Earth, and chloride ions at infinite dilution in water. This microscopic quantity is the key to understand the NMR relaxation of quadrupolar ions, such as ${ }^{7} \mathrm{Li}^{+},{ }^{23} \mathrm{Na}^{+},{ }^{25} \mathrm{Mg}^{2+},{ }^{39} \mathrm{~K}^{+}$, or ${ }^{137} \mathrm{Cs}^{+}$. Specifically, we analyzed the effect of the ionic charge and size on the distribution of the EFG tensor and on its autocorrelation function. The instantaneous EFG tensor can be reasonably well described by the Gaussian isotropic model of Czjzek et al. and the variance of the EFG induced by the surrounding solvent depends primarily on the distance of water molecules to the ions. The decay of the EFG-ACF occurs approximately in two steps, with a fast process on the 10-100 fs time scale contributing to most of the decay followed by a slower one on the 1 ps time scale. The main contribution to the integral of the EFG-ACF, to which the NMR relaxation time is proportional, is in fact this second process. In the case of the lithium cation, oscillations of the EFG-ACF correspond to the rattling of this light ion in its solvation cage. 
The first solvation shell plays the dominant role in the fluctuations of the EFG, all the more that the ion is small and charged. We proposed an extremely simple model of the charge distribution around the ion, whereby all the molecules in the first solvation shell are represented by radial dipole with an identical magnitude. The effect of the ionic size and charge on the EFG can be understood by considering the effect of the corresponding electric field on the water molecules surrounding the ion. This radial dipole model is less relevant in the case of the chloride anion, as expected. Our analysis demonstrates that the EFG-ACF, hence the NMR relaxation time, reflects primarily the collective translational motion of water molecules in the first solvation shell of the cations. Other contributions, such as further molecules or the orientation of water molecules in the first solvation shell play a less important role.

The molecular simulation approach followed in the present work provides a microscopic route to the quantitative interpretation of NMR relaxation measurements. Conversely, such experiments can thus shed new light on the debate on the molecular structure and dynamics around ions ${ }^{62-65}$ by complementing other experimental techniques such as neutron scattering or infrared, Raman and $\mathrm{THz}$ spectroscopies. ${ }^{66-69}$ Our findings also open the way to the design of improved continuous theories for NMR relaxation for small ionic solutes, which should focus on water density fluctuations around the ion. Accurate simplified models are indeed essential for the extension of the present approach to complex systems involving larger length- and longer time scales such as ions in the vicinity of biological molecules or confined in anisotropic materials such as clays. ${ }^{70-74}$ In such systems, the extreme narrowing assumption may break down due to the long time scales involved. It might then be possible to evaluate the spectral density at finite Larmor frequency by combining molecular simulations with coarse-grained models such as the lattice method recently introduced in the context of NMR spectroscopy. ${ }^{75}$

\section{ACKNOWLEDGMENTS}

The authors thank Anne-Laure Rollet, Guillaume Mériguet, and Pierre Levitz for insightful discussions. A.C. acknowledges financial support from UPMC.

\footnotetext{
${ }^{1}$ A. Abragam, The Principles of Nuclear Magnetism (Clarendon Press, Oxford University Press, Oxford [Oxfordshire], New York, 1983).

${ }^{2}$ J. Kowalewski, Nuclear Spin Relaxation in Liquids: Theory, Experiments, and Applications, Series in Chemical Physics Vol. 2 (Taylor \& Francis, New York, 2006).

${ }^{3}$ H. Hertz, Ber. Bunsen-Ges. 77, 531 (1973).

${ }^{4}$ H. Hertz, Ber. Bunsen-Ges. 77, 688 (1973).

${ }^{5}$ P. G. Wolynes, Annu. Rev. Phys. Chem. 31, 345 (1980).

${ }^{6}$ J.-H. Chen and S. A. Adelman, J. Chem. Phys. 72, 2819 (1980).

${ }^{7}$ J. T. Hynes and P. G. Wolynes, J. Chem. Phys. 75, 395 (1981).

${ }^{8}$ A. Chandra and B. Bagchi, J. Chem. Phys. 91, 1829 (1989).

${ }^{9}$ A. Chandra and B. Bagchi, J. Chem. Phys. 94, 2258 (1991).

${ }^{10}$ B.-C. Perng and B. M. Ladanyi, J. Chem. Phys. 109, 676 (1998).

${ }^{11}$ A. Sacco, Chem. Soc. Rev. 23, 129 (1994).

${ }^{12}$ S. Engström and B. Jönsson, Mol. Phys. 43, 1235 (1981).

${ }^{13}$ S. Engström, B. Jönsson, and B. Jönsson, J. Magn. Reson. 50, 1 (1982).

${ }^{14}$ P. Linse and B. Halle, Mol. Phys. 67, 537 (1989).

${ }^{15}$ S. Engström, B. Jönsson, and R. W. Impey, J. Chem. Phys. 80, 5481 (1984).

${ }^{16}$ J. E. Roberts and J. Schnitker, J. Phys. Chem. 97, 5410 (1993).
}

${ }^{17}$ M. Odelius, A. Laaksonen, M. Levitt, and J. Kowalewski, J. Magn. Reson., Ser. A 105, 289 (1993).

${ }^{18}$ M. Odelius, J. Phys. Chem. 98, 12108 (1994).

${ }^{19}$ M. Odelius and A. Laaksonen, Mol. Phys. 82, 487 (1994).

${ }^{20}$ M. Odelius and J. Kowalewski, J. Chem. Soc., Faraday Trans. 91, 215 (1995).

${ }^{21}$ B. Kirchner, D. J. Searles, A. J. Dyson, P. S. Vogt, and H. Huber, J. Am. Chem. Soc. 122, 5379 (2000).

${ }^{22}$ M. G. Müller, B. Kirchner, P. S. Vogt, H. Huber, and D. J. Searles, Chem. Phys. Lett. 346, 160 (2001).

${ }^{23}$ M. G. Müller, E. H. Hardy, P. S. Vogt, C. Bratschi, B. Kirchner, H. Huber, and D. J. Searles, J. Am. Chem. Soc. 126, 4704 (2004).

${ }^{24}$ J. Schmidt, J. Hutter, H.-W. Spiess, and D. Sebastiani, ChemPhysChem 9, 2313 (2008).

${ }^{25}$ K. Aidas, H. Ågren, J. Kongsted, A. Laaksonen, and F. Mocci, Phys. Chem. Chem. Phys. 15, 1621 (2013).

${ }^{26} \mathrm{~S}$. Badu, L. Truflandier, and J. Autschbach, J. Chem. Theory Comput. 9, 4074 (2013).

${ }^{27}$ A. Carof, M. Salanne, T. Charpentier, and B. Rotenberg, J. Phys. Chem. B 118, 13252 (2014).

${ }^{28}$ R. Baumert, R. Ludwig, and A. Geiger, Mol. Model. Annu. 2, 379 (1996).

${ }^{29}$ E. H. Hardy, M. G. Müller, P. S. Vogt, C. Bratschi, B. Kirchner, H. Huber, and D. J. Searles, J. Chem. Phys. 119, 6184 (2003).

${ }^{30}$ S. Tazi, J. J. Molina, B. Rotenberg, P. Turq, R. Vuilleumier, and M. Salanne, J. Chem. Phys. 136, 114507 (2012).

${ }^{31}$ L. X. Dang and T.-M. Chang, J. Chem. Phys. 106, 8149 (1997).

${ }^{32} \mathrm{CP} 2 \mathrm{~K}$ Developers Group, www.cp2k.org.

${ }^{33}$ P. P. Ewald, Ann. Phys. 369, 253 (1921).

${ }^{34}$ J.-P. Ryckaert, G. Ciccotti, and H. J. Berendsen, J. Comput. Phys. 23, 327 (1977).

${ }^{35}$ G. Ciccotti, M. Ferrario, and J.-P. Ryckaert, Mol. Phys. 47, 1253 (1982).

${ }^{36}$ S. Nosé, Mol. Phys. 52, 255 (1984).

${ }^{37}$ S. Nosé, J. Chem. Phys. 81, 511 (1984).

${ }^{38}$ G. Czjzek, J. Fink, F. Götz, H. Schmidt, J. M. D. Coey, J.-P. Rebouillat, and A. Liénard, Phys. Rev. B 23, 2513 (1981).

${ }^{39}$ F. Vasconcelos, S. Cristol, J.-F. Paul, L. Delevoye, F. Mauri, T. Charpentier, and G. L. Car, J. Phys.: Condens. Matter 25, 255402 (2013).

${ }^{40}$ A. Mukhopadhyay, A. T. Fenley, I. S. Tolokh, and A. V. Onufriev, J. Phys. Chem. B 116, 9776 (2012).

${ }^{41}$ R. A. Marcus, J. Chem. Phys. 24, 979 (1956).

${ }^{42}$ R. A. Marcus, Annu. Rev. Phys. Chem. 15, 155 (1964).

${ }^{43}$ J. Blumberger and M. Sprik, in Computer Simulations in Condensed Matter Systems: From Materials to Chemical Biology, Lecture Notes in Physics Vol. 704, edited by M. Ferrario, G. Ciccotti, and K. Binder (Springer, Berlin, Heidelberg, 2006), Vol. 2, pp. 481-506.

${ }^{44}$ J. D. Eaves, J. J. Loparo, C. J. Fecko, S. T. Roberts, A. Tokmakoff, and P. L. Geissler, Proc. Natl. Acad. Sci. U. S. A. 102, 13019 (2005).

${ }^{45}$ J. D. Eaves, A. Tokmakoff, and P. L. Geissler, J. Phys. Chem. A 109, 9424 (2005).

${ }^{46}$ P. L. Geissler, C. Dellago, D. Chandler, J. Hutter, and M. Parrinello, Science 291, 2121 (2001).

${ }^{47}$ B. Reischl, J. Köfinger, and C. Dellago, Mol. Phys. 107, 495 (2009).

${ }^{48}$ J. Noah-Vanhoucke and P. L. Geissler, Proc. Natl. Acad. Sci. U. S. A. 106, 15125 (2009).

${ }^{49}$ A. J. Ballard and C. Dellago, J. Phys. Chem. B 116, 13490 (2012).

${ }^{50}$ See supplementary material at http://dx.doi.org/10.1063/1.4935496 for the statistics of the EFG on the oxygen and hydrogen of water in neat water (shown in Figure S1).

${ }^{51}$ H. K. Shin, C. Kim, P. Talkner, and E. K. Lee, Chem. Phys. 375, 316 (2010).

${ }^{52}$ A. C. Brańka, D. M. Heyes, and G. Rickayzen, J. Chem. Phys. 135, 164507 (2011).

${ }^{53} \mathrm{~J}$.-B. d'Espinose de Lacaillerie, C. Fretigny, and D. Massiot, J. Magn. Reson. 192, 244 (2008).

${ }^{54}$ S. Koneshan, J. Rasaiah, R. M. Lynden-Bell, and S. H. Lee, J. Phys. Chem. B 102, 4193 (1998).

${ }^{55}$ S. Koneshan, R. M. Lynden-Bell, and J. C. Rasaiah, J. Am. Chem. Soc. 120, 12041 (1998).

${ }^{56}$ J. Bosse, D. Quitmann, and C. Wetzel, Phys. Rev. A 28, 2459 (1983).

${ }^{57}$ B. J. Alder and T. E. Wainwright, Phys. Rev. A 1, 18 (1970).

${ }^{58}$ X. Song, D. Chandler, and R. A. Marcus, J. Phys. Chem. 100, 11954 (1996).

${ }^{59}$ B. Hribar, N. T. Southall, V. Vlachy, and K. A. Dill, J. Am. Chem. Soc. 124, 12302 (2002).

${ }^{60}$ W. Kunz, Curr. Opin. Colloid Interface Sci. 15, 34 (2010).

${ }^{61}$ M. D. Baer and C. J. Mundy, Faraday Discuss. 160, 89 (2013).

${ }^{62}$ G. Stirnemann, E. Wernersson, P. Jungwirth, and D. Laage, J. Am. Chem. Soc. 135, 11824 (2013). 
${ }^{63}$ D. Laage and J. T. Hynes, J. Phys. Chem. B 112, 14230 (2008).

${ }^{64}$ D. Laage, G. Stirnemann, and J. T. Hynes, J. Phys. Chem. B 113, 2428 (2009).

${ }^{65}$ D. Laage, J. Phys. Chem. B 113, 2684 (2009).

${ }^{66}$ H. J. Bakker, Chem. Rev. 108, 1456 (2008).

${ }^{67}$ S. T. van der Post, S. Scheidelaar, and H. J. Bakker, J. Mol. Liq. 176, 22 (2012).

${ }^{68}$ S. Funkner, G. Niehues, D. A. Schmidt, M. Heyden, G. Schwaab, K. M. Callahan, D. J. Tobias, and M. Havenith, J. Am. Chem. Soc. 134, 1030 (2012).

${ }^{69}$ J. Savolainen, S. Ahmed, and P. Hamm, Proc. Natl. Acad. Sci. U. S. A. 110, 20402 (2013).
${ }^{70}$ L. Nordenskiold, D. K. Chang, C. F. Anderson, and M. T. Record, Biochemistry 23, 4309 (1984).

${ }^{71}$ P. Porion, A. M. Faugère, and A. Delville, J. Phys. Chem. C 112, 9808 (2008).

${ }^{72}$ P. Porion, A. M. Faugère, and A. Delville, J. Phys. Chem. C 113, 10580 (2009).

${ }^{73}$ P. Porion, L. J. Michot, F. Warmont, A. M. Faugère, and A. Delville, J. Phys. Chem. C 116, 17682 (2012).

${ }^{74}$ P. Porion, A. M. Faugère, and A. Delville, J. Phys. Chem. C 117, 26119 (2013).

${ }^{75}$ C. Merlet, A. C. Forse, J. M. Griffin, D. Frenkel, and C. P. Grey, J. Chem. Phys. 142, 094701 (2015). 